\title{
Analysis of hot spots in Internet Economy research based on bibliometrics abstract
}

\author{
Qian Kun $^{1, \mathrm{a}}$, Lv Yuenan ${ }^{1, \mathrm{~b}}$, Li Yingxue ${ }^{1, \mathrm{c}}$ \\ ${ }^{1}$ School of Management, Dalian Polytechnic University, DLPU, Dalian China
}

\begin{abstract}
In this paper, the core journals and CSSCI of CNKI are selected as the database, and the research overview of Internet economy is analyzed through Citespace visual software. The analysis contents mainly include publishing institutions, authors and keywords. The research found that the number of literatures on the topic of Internet economy grew rapidly, and the institutions with large publication volume mainly distributed in Beijing, Shanghai and other places.Research hotspots mainly include Internet finance, artificial intelligence and other hot words closely related to us.
\end{abstract}

\section{Introduction}

Internet economy is a new economic phenomenon with network information technology as the carrier, and has become the backbone force in the current economic and social development ${ }^{[1]}$. Mainly based on the "Internet $+"$ platform, it will integrate the Internet with traditional industrial economy and accelerate the transformation, upgrading and innovative development of traditional economy. At present, the deep integration of the Internet with various fields of economy and society has spawned many new things, which to a large extent have an impact on company production, industrial upgrading, human life, social management and other aspects. For example, the development of emerging service industries such as ecommerce and Internet finance has greatly increased the proportion of the tertiary industry and promoted the optimization and upgrading of the industrial structure. Therefore, the Internet economy, as a new driving force for China's economic development, has an important impact on the development of multiple industries and fields in social production, and is of great significance in accelerating the formation of the new normal of the economy. Therefore, in the context of Internet economy becoming a research hotspot, the current research status and progress are sorted out to provide reference for future research. Based on this, this paper uses CNKI and bibliometrics software to conduct an in-depth analysis of the literature on Internet economy, and forecasts the future development direction of Internet economy.

\section{DATA SOURCES AND METHODS}

CNKI is currently the most widely used and comprehensive data source platform for domestic scholars. It collects all kinds of journal papers, dissertations, patent yearbooks and other statistical data. Therefore, this paper takes the core journals and CSSCI of CNKI with high academic level and theoretical value as the data sources. First of all, through the retrieval of the subject word "Internet economy", a total of 520 journal literatures were obtained, and the complete retrieval time span was from 1997 to 2019. Secondly, in order to ensure the validity of the data, 520 literatures were screened. Reports, conference reviews and other literatures were manually eliminated, and 476 valid literatures were finally obtained as the data source for the mapping analysis of Internet economy research. Finally, CiteSpace5.6 visual analysis tool was used to analyze the institution and author's publication, co-occurrence and cooperation, keyword distribution and clustering, etc.

CiteSpace is a visual application software used in scientific literature to predict new trends and dynamics of scientific development. At present, CiteSpace is widely used in research progress and frontier analysis of research topics in many fields. For example, scientific research management, business economics, computer software and computer applications and other major fields. CiteSpace visual analysis plays an important role in quickly and scientifically mastering the core of the research field, sorting out the research progress, and assisting in the thesis proposal.

\section{STATISTICAL RESULTS AND ANALYSIS}

\subsection{Internet economy research time characteristic analysis}

The time characteristics of the number of literatures can fully reflect the origin and development process of the research topic, and the time characteristics of authors can

aqiankund1@163.com b1_xg91688@163.com c2393213813@qq.com 
reflect the research focus of researchers. The number of literatures published and the number of authors in Internet economy research is shown in Fig 1. On the whole, the growth trend of the number of published articles from 1997 to 2015 was basically consistent with that of the number of authors. However, since 2015, the growth trend of the number of published articles has been flat, which may be caused by the decrease in the number of published articles among authors and the increase in the number of individual published articles. Judging from the number, document number from 1997 to 2002 and the author number of peaks, 2002-2012, were identificated and are in fluctuation change, the author number after 2013 were identificated began to rapidly increase, increase obviously, only 2016 to 2017 , the phenomenon of a small slide, but the future is expected to post number number and the author will continue to increase. Therefore, with the rapid development of science and technology, more and more researchers will pay attention to the Internet economy.

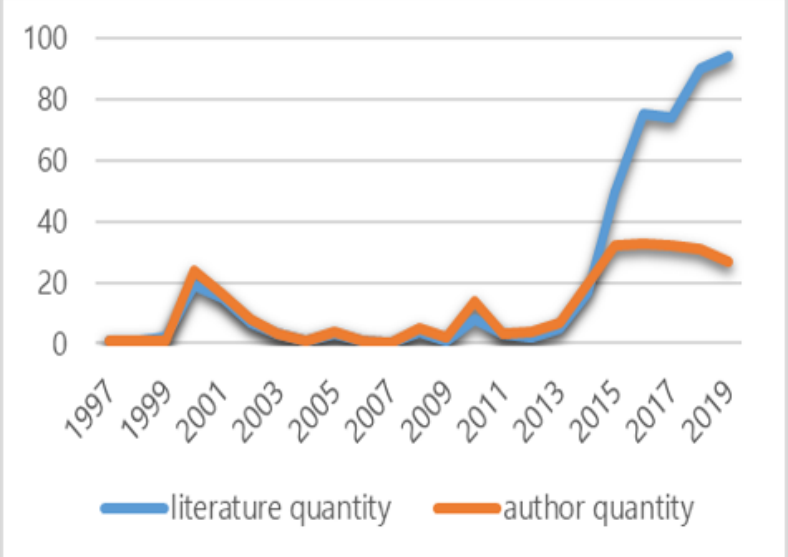

Fig1. Trend chart of literature and number of authors

\subsection{Internet Economy Research Institute Analysis}

In this paper, statistics are made on the top 10 institutions of Internet economy research, as shown in Tab I. It can be seen that Renmin University of China published the most papers (27 papers), followed by the Chinese Academy of Social Sciences (22 papers). The two institutions published far more papers than other institutions of higher learning.In addition, the publication volume is relatively more Shanghai Jiaotong University, Beijing University of Information Science and Technology, Central University of Finance and Economics, Shanghai University of Engineering Technology, Beijing Technology and Business University, etc. From the perspective of geographical distribution, the top ten universities are mainly concentrated in Shanghai and Beijing, which is closely related to the strong university lineup and the gathering of top talents. According to statistics, China's top 100 Internet companies are located in Beijing (31), followed by Shanghai (19). Therefore, the cultivation of sophisticated Internet talents can effectively promote the economic development of local Internet industry.

Table1. Top 10 institutions of Internet Economy Research publication volume

\begin{tabular}{|l|l|l|}
\hline Rank & Institute & Count \\
\hline 1 & Renmin University of China & 27 \\
\hline 2 & Chinese Academy of Social Sciences & 22 \\
\hline 3 & Shanghai Jiaotong University & 8 \\
\hline 4 & Beijing Information Science and Technology University & 7 \\
\hline 5 & Central University of Finance and Economics & 6 \\
\hline 6 & Shanghai University Of Engineering Science & 5 \\
\hline 7 & Beijing Technology and Business University & 5 \\
\hline 8 & Southwest University of Political Science and Law & 4 \\
\hline 9 & Shanghai University of Finance and Economics & 3 \\
\hline 10 & Beijing Jiaotong University & \\
\hline
\end{tabular}

\subsection{Internet Economy Research Author Analysis}

As shown in Tab II, the top five representative authors are Wang Huanchen and Huang Wenbo from Shanghai Jiao Tong University. Liu Qingzhen, Beijing University of Information Technology; Chen Bing, Nankai University; Zhang Yonglin, Beijing Normal University. Among them, Professor Wang Huanchen, who has published the most papers, first published "Network Economic Relations from the Perspective of System Science" in Soft Science in 2000, co-authored by Huang Wenbo, which has been cited for 4 times. Since the Internet was put into commercial use in 1993, it has exerted a rapid impact on the economic and social fields, and the network economy has also emerged. Therefore, Professor Wang huanchen's article has laid the foundation for today's Internet economy research. It can be seen from Fig 2 that in the early stage of Internet economy research, there were many cooperative articles among authors, such as 6 articles jointly published by Wang Huanchen, Huang Wenbo and Hou Heyin. However in recent years, the output of independent publications by researchers such as Zhang Yonglin and Liu Qingzhen has been relatively high. 


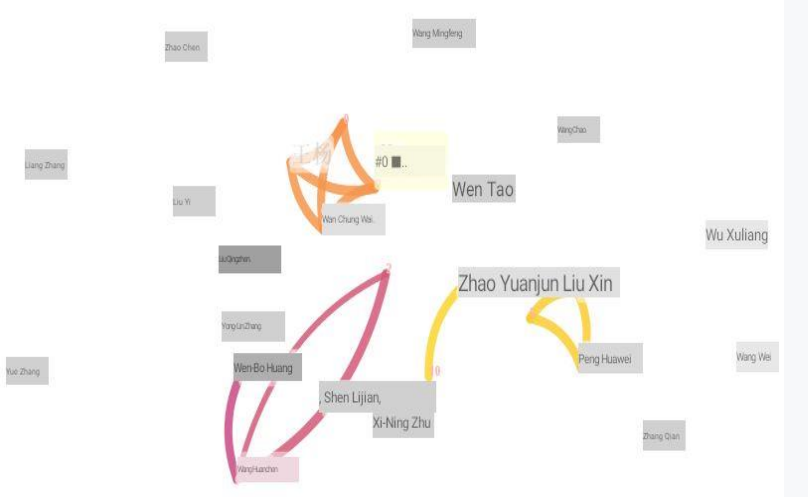

Fig2. Visual atlas of Internet Economy research authors

Table2. Top five authors of Internet Economy Research

\begin{tabular}{|c|c|c|c|}
\hline Rank & Count & Author & Year \\
\hline 1 & 6 & Wang Huanchen & 2000 \\
\hline 2 & 5 & Liu Qingzhen & 2015 \\
\hline 3 & 4 & Chen Binng & 2018 \\
\hline 4 & 4 & Huang Wenbo & 2000 \\
\hline 5 & 2 & Zhang Yonglin & 2016 \\
\hline
\end{tabular}

\subsection{Analysis of Hotspots in Internet Economy Research}

Keywords are a high summary of the core content of literature research ${ }^{[2]}$. This paper can effectively identify the hot research topics in this field through the construction and analysis of the co-occurrence map of keywords related to "Internet economy". It can be seen from Tab III and Fig 3 that the high-frequency keywords in the literature related to Internet economy from 1997 to 2019 mainly include: Internet finance, sharing economy, economic growth, e-commerce, business model, etc. Therefore, the research hotspots of Internet economy are summarized as follows:

1) Sharing economy. As a new business model under the background of "Internet +", sharing economy can realize efficient and accurate matching of idle elements, maximize the creation of surplus value, and have the advantages of low transaction costs and high resource utilization ${ }^{[3]}$. However, with the rapid development of sharing economy, it is also faced with a series of tests, for example, problems such as administrative monopoly and brutal merger and acquisition caused by regulators' dereliction of duty and abuse of power. Therefore, while the sharing economy model is respected and recognized by the public, it is crucial to innovate the regulatory forms in its development process.

2) Internet finance. Internet finance relies on big data and cloud computing to form functional service formats and service systems on an open Internet platform. At present, Internet finance has been extended to finance, lending, insurance, crowdfunding, consumer finance and other fields ${ }^{[4]}$. At the same time, it is fully involved in mainstream financial forms such as banking, securities and insurance, and gradually forms a new financial form of "Internet + technology + finance" through the application of fintech. This is of great significance to the sustainable development of China's economy. Although Internet finance has brought universal benefits to our life, economic crimes based on Internet finance innovation also come along, posing a certain threat to the development of the industry.

3) Artificial intelligence. With the continuous progress of science and technology, the "Internet $+\mathrm{AI}$ " model has become a new economic growth point, promoting the development of emerging industries. For example, in recent years, the emerging smart endowment, smart medical treatment and smart city are all products of "Internet + " artificial intelligence ${ }^{[5]}$. The emerging model of "Internet plus" artificial intelligence has brought new vigor and vitality to the development of traditional enterprises. But at the same time, artificial intelligence can replace part of the labor force, resulting in social unemployment rate increase, and other problems.

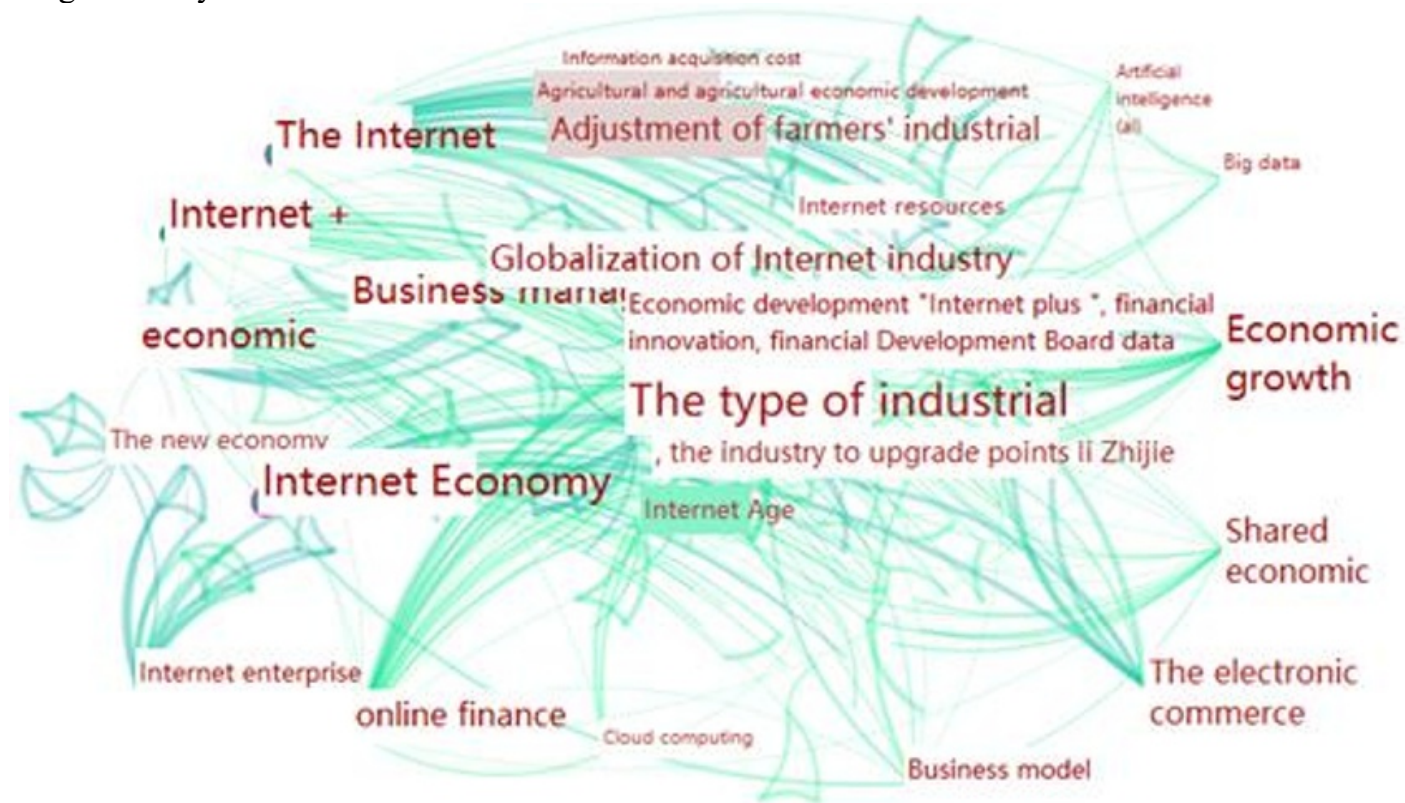

Fig3. Visual map of key words of Internet economy 
Table3. Top 10 high frequency, high centrality keywords

\begin{tabular}{|c|c|c|c|}
\hline Rank & Keywords & Frequency & Centrality \\
\hline 1 & Internet economy & 81 & 0.21 \\
\hline 2 & Internet & 72 & 0.32 \\
\hline 3 & Internet + & 44 & 0.11 \\
\hline 4 & economy & 36 & 0.40 \\
\hline 5 & Internet finance & 33 & 0.19 \\
\hline 6 & Sharing economy & 24 & 0.04 \\
\hline 7 & Economic growth & 21 & 0.05 \\
\hline 8 & Innovate & 19 & 0.18 \\
\hline 9 & Bussiness model & 9 & 0.01 \\
\hline 10 & Big data & 7 & 0.03 \\
\hline
\end{tabular}

\section{Conclusion and prospect}

Using Citespace as a tool, this paper makes a statistical analysis of literatures related to Internet economy, and draws the following conclusions: From the perspective of regional distribution of publishing institutions, research results of universities in Beijing, Shanghai and some southwest regions are in the majority; from the perspective of the authors, Wang Huanchen published the most articles and had cooperators, while the cooperation between other authors was relatively small. From the visual results of keywords, hot researches on Internet economy mainly include "Internet finance", "sharing economy", "artificial intelligence", etc. They are both derivatives of Internet economy and conducive to the further development of Internet economy.

Nowadays, the wave of Internet economy has covered all walks of life in social development, which is not only an opportunity, but also a challenge. In the process of the development of Internet and traditional industry integration, how to strengthen the related departments of supervision, regulating the behavior of regulators, how to effectively improve the relevant laws and regulations policy, how to effectively reduce the Internet's negative influence on the social and economic problems is not only the relevant government departments need to think about problems, also need to focus on key problems in the research of scholars. Therefore, using visual analysis software to explore the Internet economy research field can provide a direction for its future development.

\section{References}

1. $\mathrm{Xu}$ Xingxing. Construction and Demonstration of Internet Economic Development Evaluation Index System in China [J/OL]. Statistics and Decisionmaking,2020(11):54-57

2. Lu Lin, LIU Yangming. Research context and Evolution of Chinese Farmers' Professional Cooperatives -- Visualization Research based on CNKI core journals and CSSCI data from 1999 to 2019 [J]. Journal of Southwest University for Nationalities (Humanities and Social Sciences edition), 2020 .
3. Kong Lingying, Li Yuantong. Research on business Model of Rural Sharing Economy under the Background of Internet -- Based on Multi-Case Analysis [J]. Agricultural Economy, 2019(12).

4. Sun Yu-chen. Transformation of Supervision Mode of Internet Sharing Economy: Toward competitionoriented Supervision [J]. Hebei Science Of Law, 2018, 36(10):19-36.

5. Liao shengwu, xue yunlian, tan bihui, li siyuan, Chen baoan, liang youli, xu jun. Intelligent hospital diagnosis and treatment management strategy in the era of "Internet + " artificial intelligence [J]. Chinese hospital management,2019,39(10):5-8. 